\title{
Infrared Monitoring of Nuclear Power in Space
}

\section{David W. Hafemeister"}

This paper assesses the level of confidence for monitoring a ban on nuclear power in earth orbit using unclassified information from astronomical observatories based on Maui, Hawaii, and from the Kuiper Airborne Observatory. It is concluded that existing military and astronomical observatories can detect and identify operating nuclear reactor sources, such as the Soviet RORSAT and US SP-100, on satellites with a very high level of confidence out to distances beyond geosynchronous orbit. The smaller radioisotope thermal sources, RTG and DIPS, could be detected with a medium level of confidence under certain conditions.

\section{INTRODUCTION}

This paper analyzes our ability to detect and identify nuclear power sources in earth orbit with infrared monitoring systems.

The US is considering obtaining nuclear power from three systems: the radioisotope thermal generator (RTG), the dynamic isotope power system (DIPS), and the proposed SP-100 system, a nuclear reactor. In addition, the US is considering multi-megawatt systems and burst-power systems. The Soviet Union already has nuclear power reactors in its Radar Ocean Reconnaissance Satellites (RORSATs).

The rejected heat from these nuclear power sources is considerable

a. Center for International Security and Arms Control. Stanford University and Physics Department, Callfornia Polytechnic State University

This paper was written under the auspices of the Cooperative Research Project on Arms Reductions, a joint project of the Federation of American Sclentists and the Committee of Soviet Scientists for Peace and Against the Nuclear Threot 
Table 1: The Rejected Heat from Nuclear Power Sources

$\begin{array}{lccccc}\begin{array}{c}\text { Electrical } \\ \text { Power }\end{array} & \text { Efficiency } & \begin{array}{c}\text { Heat } \\ \text { Rejected } \\ k W\end{array} & \% & \begin{array}{c}\text { Radiator } \\ \text { Area }\end{array} & \begin{array}{c}\text { Radiator } \\ m^{2}\end{array} \\ \text { Temperature } \\ \text { RTG } & 0.3 & 7 & 4.1 & 1.2 & 500 \\ \text { DIPS } & 6.0 & 24 & 19.0 & 25-48 & 330 \\ \text { SP-100 } & 100.0 & 4 & 2,400.0 & 90 & 800 \\ \text { RORSAT* } & 5-10 & 4-10 & 100 & & 850\end{array}$

Source: SDI, Technology. Sumtuablity, and Software Washington DC: Office of Technology Assessment 1988), except 'G.L. Bennett, A Look of the Soviet Nuclear Power Program Washington DC: NASA. 1989).

(see table 1). As a result, the infrared signatures of the present generation of nuclear power plants in earth orbit are already easily observable, as will be shown below.

We will consider, in particular, the capabilities of the US Air Force Maui Optical Station (AMOS), based on Mt. Haleakala, Maui, Hawaii, at an altitude of 3,000 meters $(10,000$ feet).

Below we discuss:

- Characteristic features of the IR signature

- IR temperature determinations

- IR signatures for various nuclear power sources

- Land-based IR monitoring systems

- Countermeasures to IR monitoring

- Analysis of IR monitoring of nuclear power in earth orbit

- Conclusions

In the appendix, we consider infrared monitoring systems based on high-altitude aircraft, on satellites in low earth orbit, on satellites in geosynchronous orbit, on inexpensive sounding rockets (for a close look), and on the space shuttle. 


\section{CHARACTERISTIC FEATURES OF THE INFRARED SIGNATURE}

The generation of electricity in space, using either the thermoelectric effect .or traditional thermodynamic heat-engine cycles, requires the rejection of waste heat.

For a black-body radiator, Planck's laws describe the dependence of the spectrum and intensity of the radiation on temperature.

According to Planck's radiation law, the spectral radiant emittance of a black body is given by

$$
W_{\lambda}=\frac{e_{\lambda} C_{1}}{\lambda^{5}\left(\exp \left[C_{2} / \lambda T\right]-1\right)}
$$

where $W_{\lambda}$ is in $W / \mathrm{cm}^{2}$ per micron of spectral wavelength, $\lambda$ is the wavelength in microns $\left(1 \times 10^{-6}\right.$ meters), $T$ is the temperature in kelvins, $C_{1}$ is $3.74 \times 10^{4}, C_{2}$ is $1.44 \times 10^{4}$ and $e_{\lambda}$ is the emissivity at the wavelength $\lambda$ to account for nonideal radiators. ${ }^{1}$

The black-body spectrum peaks, according to Wien's law, at the wavelength (energy)

$$
\lambda_{\max }=\frac{2,897}{T} \quad\left(E_{\max }=\frac{1.24}{\lambda_{\max }}\right)
$$

where the energy $E_{\max }$ is in electron volts and $\lambda_{\max }$ is again in microns. Integrating over all wavelengths gives the radiant emittance,

$$
W=e s T^{*}
$$

where $W$ is in $W / \mathrm{cm}^{2}, s$ is the Stefan-Boltzmann constant $\left(=5.67 \times 10^{-12}\right.$ $\left.\mathrm{W} / \mathrm{cm}^{2}-\mathrm{K}^{4}\right)$, and $e$ is the averaged emissivity.

The radiant flux $F$ at the detecting system for a spherical radiator of surface area $A$ in square centimeters is

$$
F=\frac{W A}{4 \pi R^{2}}
$$

where $R$ is the distance to the detector. This value, however, is reduced by atmospheric absorption.

The rate of photon detection, $N$, after taking into account atmospheric absorption can be approximated by 


$$
N=\frac{F Q f \mathrm{\Gamma} A_{\mathrm{a}}}{E}(\text { counts } / \mathrm{sec})
$$

where $Q$ is the quantum efficiency of the detector (usually between 20 percent and 50 percent), $f$ is the fraction of the radiation within the detector's absorption band, $\Gamma$ is the transmissivity of the atmosphere, $A_{\alpha}$ is the area of the detector, and $E$ is the average energy of the photons in the band. Avalanche diodes can be used to detect individual infrared photons, but, at higher counting rates, photovoltaics or photoconductors that integrate the charge are used.

Because the background corrections for infrared radiation dominate when looking through the atmosphere or when looking against the earth's background, we will not attempt to determine signal-to-noise ratio directly, but will use instead the measured sensitivity functions for various existing infrared facilities to determine the detectability of power sources in space.

\section{IR TEMPERATURE DETERMINATIONS}

There are several methods that could be used to determine the temperature of radiators in space. One is to observe the peak in the black-body radiation and then to use Wein's law (equation 2 above) directly to determine the temperature. For example, if the distribution peaked at 10 microns, this would imply a temperature of $290 \mathrm{~K}$, while a peak at 4 microns would imply a temperature of $725 \mathrm{~K}$. The RTG, the DIPS, and the SP-100 have radiator temperatures that are approximately within this range (see table 1). Since the temperature for the remainder of most spacecraft is about $300 \mathrm{~K}$, the thermal radiators for heat rejection must have a much larger surface area for Wein's law to be used without complications.

Alternatively, one could make several measurements of the spectral distribution with multispectral detectors and fit the data to the Planck formula to determine the temperature. (However, if the thermal radiators are relatively small, or if their temperature is close to $300 \mathrm{~K}$, then the subtraction of the normal radiant power of the spacecraft from that of the radiators must be very accurate.) As an example, consider very-narrow- 
band detectors at 4 microns and 10 microns. The ratio of the intensity (per interval of wavelength) at 4 microns to that at 10 microns varies by a factor of 77 as the radiator's temperature is raised from $300 \mathrm{~K}$ to $800 \mathrm{~K}$. At $300 \mathrm{~K}$, the ratio is 0.072 and at $800 \mathrm{~K}$ it is 5.5 (figure 1).

Because of the great sensitivity of the intensity ratio to temperature, it is not surprising that infrared imaging technologies are capable, in theory, of measuring temperatures to an accuracy of 0.1 or $0.01 \mathrm{~K}$. However, actual measurements are more complicated for two reasons. First, the various backgrounds must be accurately subtracted so that the intensity values are specific to the object being measured. Second, the finite band-width of the detectors reduces the sensitivity of the ratio. As shown in figure 2 , a body at $800 \mathrm{~K}$ radiates 32 percent of its energy in the 3-5micron band, and 17 percent in the $8-13$-micron band. ${ }^{2}$ The intensity ratio
becomes

$$
\frac{I_{s_{\mu-S \mu}}}{I_{s_{\mu-13 \mu}}}=\frac{32 \text { percent }}{17 \text { percent }}=1.9
$$

or considerably less than the ratio of 5.5 for the very-narrow-band detectors. Nevertheless, infrared thermography from space can determine temperatures on the earth to an accuracy of about 1 kelvin.

A third way of measuring the temperature is by determining the total power radiated by a system of a known surface area and orientation, and apply equation 3 to determine the temperature.

Of these three methods, the use of several narrow-band detectors/filters to determine ratios is likely to give the most accurate determination of the temperature. By using three measurements, giving two ratios, one can make "color-color" plots to improve the analysis.

\section{NUMERICAL VALUES OF IR SIGNATURES}

Starting with the area of the thermal radiators for the SP-100, DIPS, and RTG systems and their radiated power, the temperatures of the radiators have been calculated with equation 3 . We have also considered the case of the SP-100 reactor after it has been turned off for about 20 hours, reducing its thermal output to about 27 kilowatts (about 0.1 percent of the initial power). At that point, the radiators would have a temperature of 


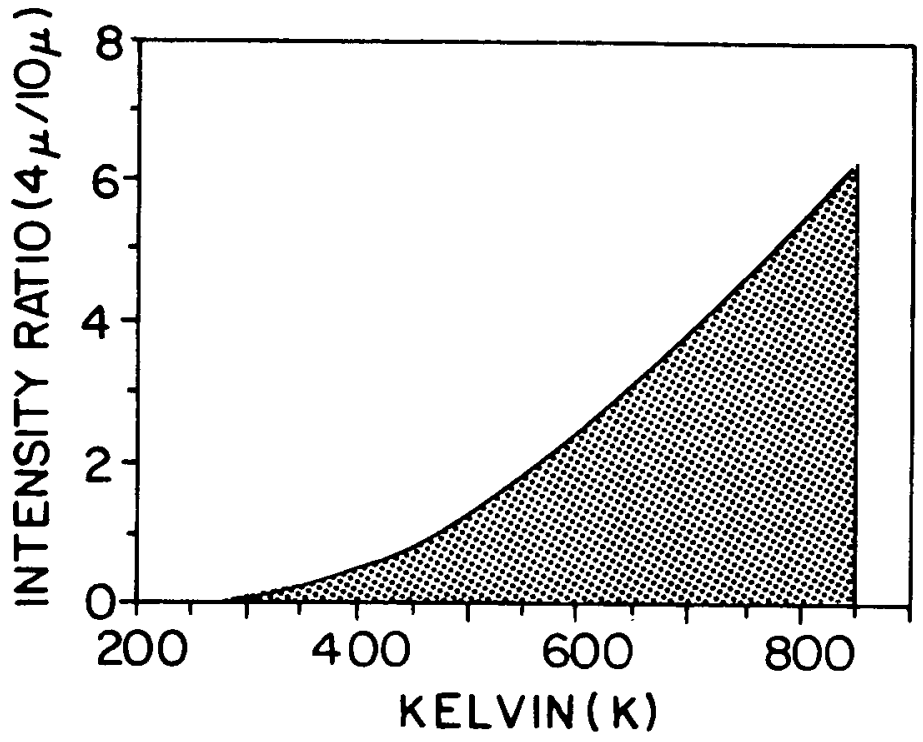

Figure 1: Intensity Ratios Determine Temperatures. The ratio of the intensity at 4 microns to the intensity at 10 microns is plotted against temperature for very-narrow-band infrared detectors.

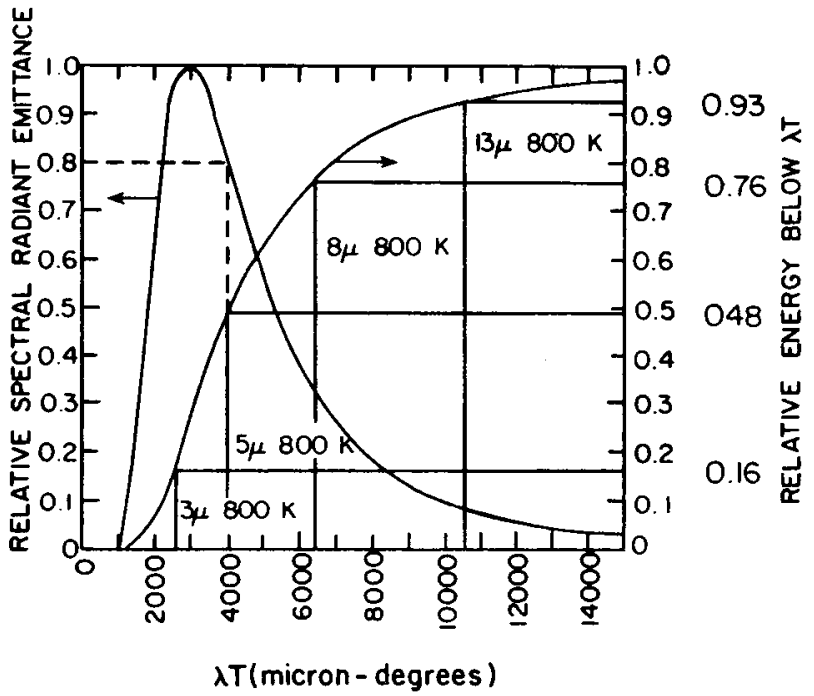

Figure 2: Finite infrared Band Detectors. The universal black-body curves plot the relative spectral emittance (left) and the integrated relative energy below $\lambda T$ (right) as a function of the product $\lambda T$. 
$270 \mathrm{~K}$-about the temperature of a spacecraft heated by sunlight alone. As a benchmark, we have also calculated the case of a sphere with a surface area of 1 square meter at $270 \mathrm{~K}$ to enable the radiation from the spacecraft itself to be calculated. This signal is similar to that from a reentry vehicle in space. Two different detection ranges have been assumed for our calculations of the radiant flux and infrared counting rates of the sensors: 300 kilometers for low earth orbit (LEO) and 40,000 kilometers for geosynchronous orbit (GEO). The results, shown in table 2, can easily be modified for other cases. ${ }^{3}$

\section{IR DETECTION SYSTEMS}

Since the parameters of military monitoring systems are classified, we use here the parameters from various infrared astronomical observatories for our calculations and conclusions. This results in an estimate of a lower bound on possible monitoring capabilities since we assume that military monitoring systems are better than those in the public sector.

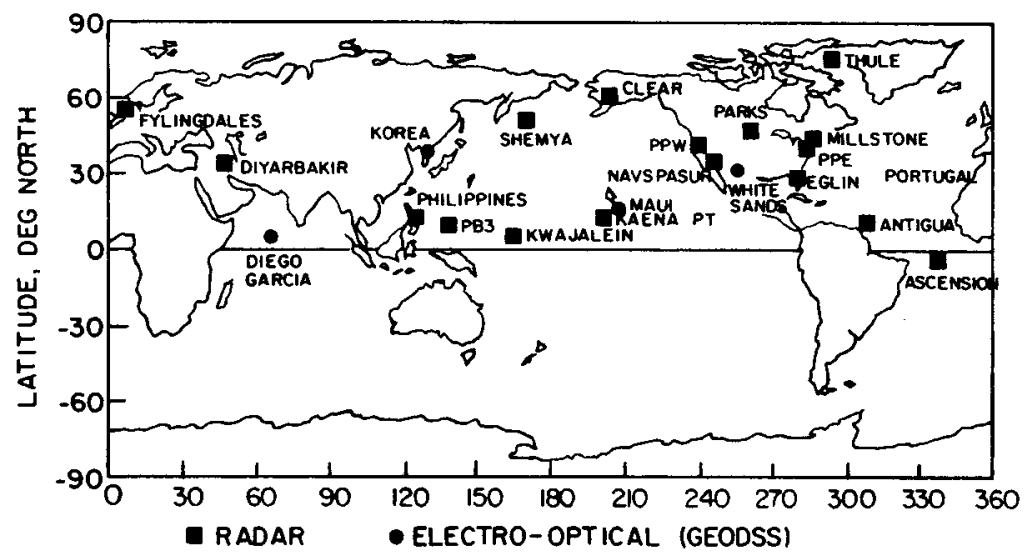

Figure 3: The GEODSS radar and electro-optical sensor locations of the GEODSS system. 


\begin{tabular}{|c|c|c|c|c|c|c|c|c|}
\hline & $\begin{array}{c}S P-100 \\
L E O\end{array}$ & $\begin{array}{l}\text { SP-100 } \\
\text { GEO }\end{array}$ & $\begin{array}{c}\text { SP-1000fft } \\
\text { LEO }\end{array}$ & $\begin{array}{l}\text { DIPS } \\
\text { LEO }\end{array}$ & $\begin{array}{l}\text { DIPS } \\
\text { GEO }\end{array}$ & $\begin{array}{l}\text { RTG } \\
\text { IFO }\end{array}$ & $\begin{array}{l}\text { RTG } \\
\text { GEO }\end{array}$ & $\underset{L E O}{\text { Benchmark }}$ \\
\hline $\begin{array}{l}\text { Electrical } \\
\text { power kW }\end{array}$ & 100 & 100 & 0 & 6.00 & 6.00 & 0.30 & 0.30 & 0 \\
\hline $\begin{array}{l}\text { Rodlated } \\
\text { power kW }\end{array}$ & 2,400 & 2,400 & 27 & 18.60 & 18.60 & 4.10 & 4.10 & 0.30 \\
\hline Area $m^{2}$ & 90 & 90 & 90 & 28.00 & 28.00 & 1.15 & 1.15 & 1.00 \\
\hline Temperature $K$ & 827 & 827 & 269 & 329 & 329 & 500 & 500 & 270 \\
\hline$\lambda_{\max } \mu$ & 3.50 & 3.50 & 10.76 & 8.82 & 8.82 & 5.79 & 5.79 & 10.71 \\
\hline Distance $\mathrm{km}$ & 300 & 40,000 & 300 & 300 & 40.000 & 300 & 40,000 & 300 \\
\hline Total flux $\mathrm{W} / \mathrm{cm}^{2}$ & $2.12 \times 10^{-10}$ & $1.19 \times 10^{-14}$ & $2.39 \times 10^{-12}$ & $1.65 \times 10^{-12}$ & $9.26 \times 10^{-17}$ & $3.63 \times 10^{-13}$ & $2.04 \times 10^{-17}$ & $2.70 \times 10^{-4}$ \\
\hline $\begin{array}{l}\text { Fraction In } \\
8 \mu-13 \mu\end{array}$ & 0.15 & 0.15 & 0.29 & 0.36 & 0.36 & 0.30 & 0.30 & 0.29 \\
\hline $\begin{array}{l}\text { Flux In } 8 \mu-13 \mu \\
W / c m^{2}\end{array}$ & $3.18 \times 10^{-11}$ & $1.79 \times 10^{-16}$ & $6.93 \times 10^{-19}$ & $5.92 \times 10^{-10}$ & $3.32 \times 10^{-17}$ & $1.09 \times 10^{-19}$ & $6.12 \times 10^{-10}$ & $7.82 \times 10^{-15}$ \\
\hline$E_{\max } \otimes V$ & 0.35 & 0.35 & 0.12 & 0.14 & 0.14 & 0.21 & 0.21 & 0.12 \\
\hline $\begin{array}{l}\text { Total photon } \\
\text { count } / s \theta c\end{array}$ & $2.26 \times 10^{13}$ & $1.27 \times 10^{\circ}$ & $7.81 \times 10^{11}$ & $4.41 \times 10^{11}$ & $2.48 \times 10^{7}$ & $6.38 \times 10^{10}$ & $3.59 \times 10^{6}$ & $8.78 \times 10^{\circ}$ \\
\hline $\begin{array}{l}\text { Stgnal intensity at } \\
w_{1} W / \mathrm{cm}^{2} \mu\end{array}$ & 0.48 & 0.48 & $5.73 \times 10^{-5}$ & $0.37 \times 10^{-4}$ & $6.37 \times 10^{-4}$ & $2.73 \times 10^{-2}$ & $2.73 \times 10^{-2}$ & $6.05 \times 10^{-5}$ \\
\hline$W_{\lambda} \times$ areo $W / \mu$ & $4.29 \times 10^{8}$ & $4.29 \times 10^{5}$ & 51.54 & 178.35 & 178.35 & 314.26 & 314.26 & 0.61 \\
\hline Diff nux $W / \mathrm{cm}^{2} \mu$ & $3.79 \times 10^{-11}$ & $2.13 \times 10^{-11}$ & $4.56 \times 10^{-16}$ & $1.58 \times 10^{-14}$ & $8.87 \times 10^{-0}$ & $2.78 \times 10^{-\mu}$ & $1.56 \times 10^{-11}$ & $5.35 \times 10^{-12}$ \\
\hline $\begin{array}{l}\text { Collected } \\
\text { energy } W / \mu\end{array}$ & $7.62 \times 10^{-7}$ & $4.29 \times 10^{-11}$ & $9.16 \times 10^{-11}$ & $3.17 \times 10^{-10}$ & $1.78 \times 10^{-4}$ & $5.59 \times 10^{-10}$ & $3.14 \times 10^{-4}$ & $1.08 \times 10^{-12}$ \\
\hline
\end{tabular}


IR Monitoring from Land-based Telescopes

The Air Force Maui Optical Station (AMOS) operates telescopes in the visible and the infrared ranges for observing satellites and re-entry vehicles as well as astronomical objects. ${ }^{4}$ The observatory is located at an altitude of 3,000 meters $(10,000$ feet); this greatly reduces absorption by water vapor and clouds. The relatively stable climate of dry air at the AMOS site gives a good astronomical seeing of about 1 arcsecond (arcs), or 5 microradians ( $\mu \mathrm{rad}$ ). Since the AMOS observatory is located at latitude 20 degrees north it can observe all satellite orbits.

The US government operates the multistation, but classified, GroundBased Electro-Optical Deep-Space Surveillance System (GEODSS). ${ }^{5}$ The location of the radar and electro-optical sensors are shown in figure $3 .^{6}$

The AMOS facility has one 1.6-meter and two 1.2-meter diameter Cassegrain telescopes that can be used for measurements in the visible and the infrared. The AMOS telescopes are set on high-performance, three-axis mounts for fast tracking. The observatory has a Beam Director/Tracker (BD/T) system. This expands a laser beam to 0.8 meters in diameter, directs the beam onto a satellite, and tracks the reflected light with a 0.8-meter observing telescope. The BD/T enables the AMOS facility to track objects with angular velocities of up to 5 degrees/sec, and accelerations of 4 degrees/ $/ \mathrm{sec}^{2}$. The $\mathrm{BD} / \mathrm{T}$ system can easily track satellites in LEO with velocities of about 0.5 degrees/sec. In addition, satellites can be tracked by using radar, by observing the diffuse scattered light from the sun or earth, or by observing their infrared emissions.

There are several similar facilities operated by the US Department of Defense (DoD) to track satellites.

\section{Visual Identification}

The AMOS facility is equipped with adaptive optics to remove the blurring effects of atmospheric turbulence. The Compensated Imaging System (CIS) adaptive-optics system is used with the 1.6-meter telescope, reducing the atmospheric seeing from 1 arcsecond to its diffraction-limited value of 0.07 areseconds ( $0.4 \mu \mathrm{radians})$ at visible wavelengths of about 0.5 microns. This improved capacity gives a resolution of 0.2 meters ( 8 inches) for a satellite at an altitude of 500 kilometers, and 0.1 meters for a satellite at an altitude of 250 kilometers. The high quality of the photographs available from this facility would allow some visual identification of the 
properties of a satellite. The advantages of CIS are greatly diminished for longer wavelength infrared radiation (see table 3).

The Cassegrain telescopes at AMOS have very long focal lengths of about 25 meters, giving a plate scale of $8 \mathrm{arcs} / \mathrm{mm}$, or $40 \mu \mathrm{rad} / \mathrm{mm}$. A large variety of arrays of infrared detectors are available, and very large arrays, with perhaps as many as 40,000 pixels, are in the planning

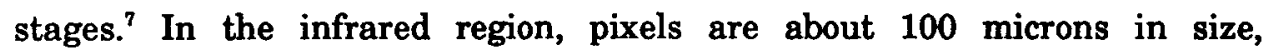
corresponding to an angular spread of 0.8 arcseconds (4 $\mu$ radians). In the visible region, pixels are about 10 microns in size, corresponding to 0.08 arcseconds (0.4 $\mu$ radians); therefore, smaller sized pixels will not improve the resolution. When combined with a diffraction grating to spread out the spectrum, one can obtain a measurement of spectra as a function of distance along an extended body if it is large enough.

\section{Spectral Measurements}

Background can be subtracted in two ways. One approach is to shake or wobble the secondary mirror of the Cassegrain telescopes, spending almost 50 percent of the time on the object, and almost 50 percent of the time on the neighboring infrared background. These background corrections can be done with great accuracy; it is possible to observe a signal that is about one part in a million of the background. Alternatively, by using a large infrared array, one can simultaneously collect the background and the signal in different parts of the array, and then subtract the background directly. The AMOS facility can measure the following limits, or lower bounds, with a signal-to-noise ratio of 1 (one standard deviation) after background subtraction with a one second integration time: ${ }^{8}$

Table 3: Resolution of the 1.6 meter AMOS CIS as a Function of Wavelength

$\begin{array}{ccc}\lambda & \text { Angular resolution } & \text { Linear }(500 / 250 \mathrm{~km}) \\ \mu & \text { arcs }(\mu \mathrm{rad}) & \mathrm{m} \\ 0.5 & 0.07(0.4) & 0.2 / 0.1 \\ 4.0 & 0.60(3.00) & 1.6 / 0.8 \\ 10.0 & 1.50(7.50) & 4.0 / 2.0 \\ & & \text { Source: author }\end{array}$


$3-4$ micron band, limit of $1 \times 10^{-17} \mathrm{~W} / \mathrm{cm}^{2}$

8-13 micron band, limit of $5 \times 10^{-18} \mathrm{~W} / \mathrm{cm}^{2}$

These two windows have been chosen because the sky radiance is very small in these regions (about 50 microwatts $/ \mathrm{cm}^{2}$ ). ${ }^{9}$

Longer integration times would enhance the data. For example, a 4-second integration time would give a signal-to-noise ratio of 2 . A satellite in LEO can be measured for about 4 minutes (240 seconds), but in practice the improvement is not as large as the square root of 240 because of the variations in the atmospheric background.

Given 30 minutes of uninterrupted observation of a satellite in high or geosynchronous orbit, the AMOS facility can also split the signal, using a grating and individual pixels, into 24 narrow wavelength bands of 0.05 microns each near 10 microns, with a sensitivity of $5 \times 10^{-18} \mathrm{~W} / \mathrm{cm}^{2}$ and a signal-to-noise ratio of 1 .

\section{IR Monitoring from Satellites and Rockets}

In the appendix, we discuss the possible use of infrared observations of satellites from aircraft flying at high altitudes, satellites in low and GEOs, sounding rockets, and space shuttles.

\section{COUNTERMEASURES}

\section{Directed Radiators}

It would be possible to design a heat exchanger that preferentially radiated in a conical beam directed away from an observer's infrared sensors. In fact, the idea of such a "radiator in a waste basket" has been explored by the General Atomics Corporation (GA): ${ }^{10}$

Thermal signature control is possible by providing a thermal barrier around the reactor and limiting radiation to one direction only.

However, such a directional radiator might be discovered by one of the following approaches for the preferential radiator of GA's putative 6-kilowatt-electric reactor with a conical full-angle of about 90 degrees: 
- The infrared could be observed by satellites in higher LEO orbits (such as US reconnaissance satellites, Landsat, or SPOT) as they pass through the beam.

- The reduced efficiency of the directional radiator would increase the cross-sectional area of the satellite by about a factor of ten. This would be easily observable with observations in the visible region with an AMOStype telescope, or with closely passing US reconnaissance satellites.

- Because a reactor's gamma-ray fluxes cannot be made directional, they could be measured by gamma-ray telescopes.

- New pop-up or shuttle systems could examine the satellite on a closelook basis.

\section{Pulsed Reactors}

Pulsed reactors have been suggested for electromagnetic launchers (EMLs, or railguns), free-electron lasers (FELs), neutral particle beam (NPB) weapons and active discriminators, and the Fission Activated Laser Concepts (FALCON and Centaurus) for the Strategic Defense Initiative. These reactors might conceivably have powers as large as $\mathbf{5}$ gigawatts electric.

Consider, for example, a reactor with a power of 100 megawatts electric. If the electrical output lasted for 100 seconds, it would transmit $10^{10}$ joules. If the pulsed reactor had an efficiency of about 10 percent, it would be necessary to reject about $10^{11}$ joules of heat. In order to hide this large amount of excess heat, it would be very ineffective to heat a large mass. A more effective countermeasure would be to use a phase transition, to turn a solid into a liquid. If the large latent heat of ice was used to mask the heat, it would take about 300 tonnes of ice to hide the full signal.

Of course, the burst reactor could be made in smaller sizes and operated for shorter times, but ultimately the full-scale deployment model would have to be tested in space without being observed, and that would be hard to do because of the large thermal systems. 


\section{CONCLUSIONS}

In order to summarize the results, we will compare the fluxes calculated in table 2 for the 8-13- and 3-4-micron windows with the capabilities of the AMOS facility.

$S P-100$

The infrared signal for the 2.4-megawatt SP-100 is very large. Table 4 indicates that (for a 1-second integration time) it could still be detected at about 700,000 kilometers. This is 20 times the distance of geosynchronous orbit. The smaller $50-100 \mathrm{~kW}$ RORSATs are routinely detected and identified in LEO by AMOS.

For the SP-100, this distance is also very realistic for identification because its reactor radiates large amounts of heat at about $800 \mathrm{~K}\left(\lambda_{\max }=\right.$ 3.5 microns), well separated from the normal thermal radiation of nonnuclear satellites. We conclude that an SP-100 could be identified with very high confidence well beyond GEO.

If the SP-100 has been shut down for 20 hours, its thermal power is reduced from 2.4 megawatts to 27 kilowatts, reducing its rejection temperature to $270 \mathrm{~K}$. Because this is about the normal temperature of non-nuclear satellites, the infrared signature would not identify the reactor, and it would have to be recognized by visual means or

Table 4: Detection Ranges for the $8 \mu-13 \mu$ and $3 \mu-4 \mu$ wave bands

\begin{tabular}{|c|c|c|c|c|}
\hline & $\begin{array}{c}F_{\text {qu- }^{-13 x}} \\
W / \mathrm{cm}^{2}\end{array}$ & $\begin{array}{c}\text { Range } \\
\mathrm{km}\end{array}$ & $\underset{W / \mathrm{cm}^{2}}{\mathbf{F}_{2-4}}$ & $\begin{array}{c}\text { Range } \\
\mathrm{km}\end{array}$ \\
\hline SP-100 & $3.2 \times 10^{-11}$ & 800,000 & $3.8 \times 10^{-11}$ & 600,000 \\
\hline SP-100 off & $6.9 \times 10^{-13}$ & 100,000 & $4.6 \times 10^{-15}$ & 6,400 \\
\hline DIPS & $5.9 \times 10^{-13}$ & 100,000 & $1.6 \times 10^{-14}$ & 12.000 \\
\hline RTG & $1.1 \times 10^{-13}$ & 40,000 & $2.8 \times 10^{-14}$ & 16,000 \\
\hline Benchmark & $7.8 \times 10^{-15}$ & 10,000 & $5.4 \times 10^{-17}$ & 700 \\
\hline
\end{tabular}


interrogated by a nearby satellite with $14 \mathrm{MeV}$ neutrons to detect the fissile material in its core.

$R T G$

Because of its fainter signal, identification of a 5-kilowatt RTG in GEO would be very difficult. For the case of the RTG in LEO, the more modest temperature of $500 \mathrm{~K}$ and its much smaller reject power of 4.1 kilowatts (see table 1) would require a subtraction of its non-nuclear thermal image. It is possible to remove this background if the areas are known very accurately. A 1-square-meter area at $270 \mathrm{~K}$ radiates about 0.3 kilowatts (see table 2). The areas of spacecraft vary between about 20 and 450 square meters. If we assume areas of 20 and 100 square meters, the non-nuclear thermal signatures would then be 6 and 31 kilowatts respectively. Hence, the nuclear/non-nuclear ratios are:

$$
\begin{aligned}
& \text { for area }=20 \mathrm{~m}^{2} \quad 0.68 \\
& \text { for area }=100 \mathrm{~m}^{2} \quad 0.13
\end{aligned}
$$

In the infrared bands under discussion, these ratios become:

$\begin{array}{lcc} & 3 \mu-4 \mu & 8 \mu-13 \mu \\ \text { for area }=20 \mathrm{~m}^{2} & 26.0 & 0.69 \\ \text { for } \text { area }=100 \mathrm{~m}^{2} & 5.2 & 0.14\end{array}$

By using the additional "color" ratios and curve fitting, it is easy to develop algorithms to estimate the RTG's temperature and heat output, but clearly, the degree of confidence in this approach will depend on having good data and on knowing the heat transfer characteristics of the entire satellite.

Since satellites have predictable orbits, and since there are a number of reconnaissance satellites (for example, KH, Landsat, and SPOT) in orbit, a close encounter will certainly take place. It would be difficult for the RTG to avoid detection during the close encounter because the RTG's power derives from plutonium-238 radioactivity that cannot be turned off. One could not only measure the satellite area accurately to improve the calculation's accuracy, but, more important, one could use an infrared monitoring system (multispectral, or commercial thermal imager) to make 
a thermal image of the satellite and look for the 500-K RTG radiator.

This type of identification could be done with existing equipment and is essentially certain; but it could be frustrated by rotating the nuclear satellite's sensitive parts away from the reconnaissance satellite. It would also be possible to cover the RTG radiator with a movable cover during a brief close encounter.

We assign a medium probability of identification of an RTG source by using accurate algorithms on well-understood satellites, and we assign a high probability of identification of RTG radiators with thermal imaging (if they have not been covered) over a long period of time.

\section{DIPS}

As in the case of the RTG, it is only feasible to consider the identification of DIPS in LEO. The case is further complicated because the DIPS radiator's temperature of $330 \mathrm{~K}$ is very close to non-nuclear satellites' usual temperatures of $270-300 \mathrm{~K}$. On the other hand, the reject thermal power for the 6-kilowatt-electric DIPS is 19 kilowatts-almost five times larger than the 4.1 kilowatts of the RTG, making a larger contribution to the total radiation from the satellite. The nuclear/non-nuclear ratios of intensities for the 6-kilowatt-electric DIPS are as follows:

$\begin{array}{lccc}\text { for area }=20 \mathrm{~m}^{2} & \text { total } & 3 \mu-4 \mu & 8 \mu-13 \mu \\ \text { for area }=100 \mathrm{~m}^{2} & 3.2 & 15.0 & 3.8 \\ & 0.6 & 2.9 & 0.76\end{array}$

Our conclusions for DIPSs are similar to those for RTGs, except that an RTG would be easier to identify with a close-passing thermal imager because its temperature is higher $(500 \mathrm{~K})$ than a DIPS's $(330 \mathrm{~K})$. On the other hand, the higher thermal power of DIPS would be more noticeable if a satellite's total emitted power were being measured.

In conclusion, existing facilities based on the ground, on high-altitude aircraft, or in LEO can easily monitor and identify the infrared signatures from SP-100-class nuclear reactors based on US or Soviet satellites to beyond GEO because of the reactor's high radiator temperature and large power.

For the identification of RTGs and DIPS, however, the detection 
ranges of 40,000 and 100,000 kilometers (see table 4) must be reduced to compensate for the host satellite's normal thermal background. After background subtraction, the detection and identification of RTGs and DIPS in LEO should be possible, but with much less confidence.

\section{ACKNOWLEDGEMENTS}

I would like to thank Glenn Ashley, Lisa Ensman, Carl Gillespie, Joel Primack, Ted Postol, Frank von Hippel, and Fred Witteborn for useful comments on this work.

\section{APPENDIX}

IR Observations from High-altitude Aircraft

For the past two decades, airplanes at an altitude near 15 kilometers have used cooled infrared detectors to monitor astronomical objects. At these high altitudes, the absorption from water vapor and clouds has been essentially eliminated, and the absorption due to carbon dioxide has been substantially reduced.

The NASA Ames Research Center, Moffett Field, California, operates the Kuiper Airborne Observatory (KAO). The flying KAO observatory carries a 0.9meter-diameter telescope at altitudes up to 14 kilometers (45,000 feet). Observatory time on the $\mathrm{KAO}$ is relatively inexpensive-about $\$ 100,000$ per flight. Since the KAO can fly at any latitude, it can observe satellites in all orbits.

To first order, the one-meter KAO observatory has about the same ultimate limit in sensitivity as the AMOS facility, $5 \times 10^{-18} \mathrm{~W} / \mathrm{cm}^{2}-\mu$ at $10 \mathrm{microns}(\mathrm{S} / \mathrm{N}=$ 1). However, the $\mathrm{KAO}$ is much better than ground-based telescopes at wavelengths longer than 20 microns because of its higher altitude.

The measurements by the KAO of the infrared spectrum of the 1987 supernova were impressive." The standard deviations of the data points in the infrared spectra were $5 \times 10^{-18} \mathrm{~W} / \mathrm{cm}^{2}-\mu$ between 3 and 13 microns.

The KAO has used a $10 \times 50$ pixel array, each pixel having a size of $75 \times 75$ microns for measuring the infrared spectrum between 7 and 25 microns. There are future plans to build an airborne astronomical observatory with a three-meter mirror, the Stratospheric Observatory for IR Astronomy (SOFIA). This would improve the light-gathering ability by a factor of 10 and the resolution by a factor of 3.

The KAO is not designed to track satellites, but it has observed them as they 
pass through the stellar field. Rather than modify the KAO to observe satellites, it would be far easier to use existing DoD airborne observatories that already track satellites. Narrow spectra sensitivity of the order of $d \lambda \lambda=0.05$ percent are obtainable. ${ }^{12}$

In addition, the Department of Defense is constructing the Airborne Optical Adjunct (AOA) for tracking re-entry vehicles against the cold space background. ${ }^{13}$ Since the radiated power of an RV is only 0.01 percent of the SP-100's radiated power and 2 percent-8 percent that of the RTG and DIPS, it is clear that tracking nuclear power in space is much easier than tracking RVs.

\section{IR Monitoring from Satellites in LEO}

Satellites such as US reconnaissance satellites, Landsat, the Space Telescope, or the French SPOT satellite can obtain excellent visual images of nearby satellites.

In addition, the Landsat and SPOT satellites have multispectral capabilities that can be used to monitor the infrared signatures of satellites. Against a cold space background, these satellites can detect very small thermal signatures. For example, the Thematic Mapper uses $\mathrm{Si}, \mathrm{InSb}$ and $\mathrm{HgCdTe}$ detectors, cooled to $95 \mathrm{~K}$, to detect in the $0.45-12.5$-micron range. ${ }^{14}$ It was able to resolve angles of 9 arcseconds (43 $\mu$ radians) in the visible and near infrared, and 35 arcseconds (170 $\mu$ radians) in the 10.4-12.5-micron thermal band. This is easily sufficient for observing power sources against the cold background of space and against the warm background of the earth under certain conditions.

Longwave-infrared observations have been carried out from LEO with the Infrared Astronomical Satellite (IRAS), obtaining an angular resolution of 20 arcseconds (100 $\mu$ radians) at longer wavelengths up to 100 microns from colder sources. $^{15}$

There are a variety of US DoD satellites that could perform this monitoring task from LEO, or from somewhat higher orbits. For example, the US DoD is constructing the "Teal Ruby" infrared sensor to be placed in a satellite in LEO to track moving aircraft against the earth's background. ${ }^{16}$

If the SDIO Program gets to its second phase, it will be necessary to deploy the Space Surveillance and Tracking System (SSTS). ${ }^{17}$ The SSTS satellites would carry longwave infrared (LWIR) sensors to track RVs and post-boost vehicles (PBVs) against the cold temperature of space above the horizon of the earth. Since the RVs have a thermal image considerably smaller than nuclear power reactors in space, this approach is clearly viable for the detection of power sources but rather expensive.

\section{Infrared Monitoring from GEO}

The earth subtends an angle of about 20 degrees from GEO. Detection of power sources above the earth is a difficult task because of the infrared emissions of the 
earth and the daytime reflection of sunlight. Nevertheless, current DoD satellites at GEO are able to detect and localize the 100-megawatt plumes from the primary boosters of missiles. The American Physical Society Directed Energy Weapons Study Group has estimated that a third stage booster of 2 megawatts would be detectable from GEO. ${ }^{13}$ However, these observations of missile plumes are greatly assisted by the discrete molecular transitions in the burning process that show up between 2.5 and 4.5 microns. The black-body spectrum from a 2.4-megawatt reactor could be observed from GEO, but it would not be worth the effort: the other options are much easier.

\section{Infrared Monitoring from Pop-up Missiles}

If one side were to become very suspicious and wished to examine the potential nuclear source at a closer distance, it could launch direct-ascent sounding rockets to get a close look. The counting rates for infrared and gamma rays would be enhanced by a factor of one million if the distance of closest approach was reduced from 1,000 kilometers to 1 kilometer. If a system measures a counting rate $N$ at its closest approach $b$ and has $4 \pi$ sensitivity, the total counts for the fly-by is

$$
\pi b N / v
$$

where $v$ is the relative velocity at closest approach. Such systems have been proposed to measure infrared signals from RVs, to supplement the SSTS for the SDI program." Since the mission requirements for the rocket would not be as great as for LEO missions, the costs for this type of sounding rocket have been estimated at about $\$ 1$ million.

\section{Infrared Monitoring from a Space Shuttle}

The US space shuttle has experienced problems and cost overruns. The very large cost of a dedicated shuttle flight is prohibitive at this time. Nevertheless, a shuttle could be used for very close examination of objects in lower orbits. By flying in a close-coorbital position, the shuttle crew could use an array of passive measuring devices for infrared, gamma rays, and neutrons. In addition, the crew could also interrogate the satellite with $14 \mathrm{MeV}$ neutrons to determine its contents. In 1982 , the US DoD placed the Cryogenic Infrared Radiance Instrumentation (Cirris) payload into the space shuttle Columbia for measurements in the LWIR.20 The Cirris telescope is capable of resolutions of 20 arcseconds (100 $\mu$ radians) and has 0.5-degree field of view. 


\section{NOTES AND REFERENCES}

1. R. Hudson, Infrared System Engineering (New York: Wiley, 1969).

2. ibid.

3. The emissivity of practical thermal radiators in the infrared region is close to one. The value of the temperature is not very dependent on this choice because radiation is proportional to the fourth power of the temperature, $T^{*}$.

4. AMOS User's Manual, (Maui, Hawaii: Air Force Maui Optical Station, 1988).

5. Anti-Satellite Weapons, Countermeasures, and Arms Control, (Washington DC: Office of Technology Assessment, 1985).

6. Source: Aerospace Corporation, in OTA, 1988 op. cit.

7. SDI, Technology, Survivability, and Software (Washington DC: Office of Technology Assessment, 1988).

8. Obtained with calibrated black bodies and with the star Vega. Glenn Ashley (AMOS), private communication.

9. Infrared spectra have been taken of satellites at AMOS. See F. Witteborn, K. O'Brien, and L. Caroff, Measurement of the Nighttime Infrared Luminosity of Spacelab 1 in the $H$ - and $K$ - Bands, NASA Technical Memorandum 85972, March 1985; F. Witteborn, L. Caroff, D. Rank, and G. Ashley, Nighttime Spectroscopic and Photometric Observations of Spacelab 2 and Other Satellites, to be published.

10. R. Dahlberg, Star-C: Nuclear Power Systems for Space Exploration, (San Diego, California: General Atomics).

11. D. Rank, P. Pinto, S. Woosley, J. Bregman, F. Witteborn, T. Axelrod, and M. Cohen, Nature, 331, p.505 (1988).

12. R. Rubin, J. Simpson, E. Erickson, and M. Hass, Astrophysical Journal, 327, p.377 (1988).

13. See OTA, 1988, p.78.

14. J. Fraser in K. Tsipis, D. Hafemeister, and P. Janeway, eds., Arms Control Verification, (Washington DC: Pergamon-Brassey, 1986).

15. G. Neugebauer et al, Science, 224, p.14 (1984); G. Rieke et al, Science, 231, p.807 (1986).

16. OTA, 1988. 
17. ibid.

18. Report to the Am. Phys. Soc., 1987.

19. OTA, 1988.

20. Aviation Week and Space Technology, 14 June 1982, p.20. 